\title{
Identification and profiling of miRNAs during herbivory reveals jasmonate-dependent and -independent patterns of accumulation in Nicotiana attenuata
}

\author{
Tohir A Bozorov, lan T Baldwin and Sang-Gyu Kim
}

\begin{abstract}
Background: Plant microRNAs (miRNAs) play key roles in the transcriptional responses to environmental stresses. However, the role of miRNAs in responses to insect herbivory has not been thoroughly explored. To identify herbivory-responsive miRNAs, we identified conserved miRNAs in the ecological model plant Nicotiana attenuata whose interactions with herbivores have been well-characterized in both laboratory and field studies.

Results: We identified 59 miRNAs from 36 families, and two endogenous trans-acting small interfering RNAs (tasiRNA) targeted by miRNAs. We characterized the response of the precursor and mature miRNAs to simulated attack from the specialist herbivore Manduca sexta by quantitative PCR analysis and used ir-aoc RNAi transformants, deficient in jasmonate biosynthesis, to identify jasmonate-dependent and -independent miRNA regulation. Expression analysis revealed that groups of miRNAs and tasiRNAs were specifically regulated by either mechanical wounding or wounding plus oral secretions from $M$. sexta larvae, and these small RNAs were accumulated in jasmonate-dependent or -independent manners. Moreover, cDNA microarray analysis indicated that the expression patterns of the corresponding target genes were correlated with the accumulation of miRNAs and tasiRNAs.

Conclusions: We show that a group of miRNAs and tasiRNAs orchestrates the expression of target genes involved in N. attenuata's responses to herbivore attack.
\end{abstract}

Keywords: Anti-herbivore defense, Jasmonate, Manduca sexta, miRNA, Nicotiana attenuata, tasiRNA

\section{Background}

A group of non-coding small RNAs (smRNAs) plays an important role in transcript regulation by binding to their target sequences, resulting in transcriptional degradation, transcriptional or translational inhibition of the targets [1-5]. The smRNAs are classified into two major classes: microRNAs (miRNAs), and small interfering RNAs (siRNAs). Primary transcripts of miRNAs are processed into precursors of miRNAs that form secondary stem-and-loop structures, which are processed by the ribonuclease DICER-like 1 (DCL1) into miRNA/miRNA* duplexes, which are subsequently incorporated into the RNA induced silencing complex (RISC) [5-7]. The

\footnotetext{
* Correspondence: skim@ice.mpg.de

Department of Molecular Ecology, Max Planck Institute for Chemical Ecology, Hans-Knöll-Straße 8, Jena D-07745, Germany
}

siRNAs are further classified into trans acting siRNAs (tasiRNAs), chromatin-associated cis acting siRNAs, and natural antisense siRNAs, based on their biogenesis [810]. Biogenesis of tasiRNAs is regulated by miRNAs, which direct cleavage of primary tasiRNA (TAS) transcripts encoding tasiRNAs, resulting in second-strand RNA synthesis by RNA-dependent RNA polymerases (RdRs). The double-stranded RNAs are diced by DCL4 to generate tasiRNAs in Arabidopsis thaliana [9-11].

Plant miRNAs and siRNAs are involved in several developmental processes [12]: embryogenesis [13], organ polarity [14], leaf formation [15], root development $[11,16]$, phytohormone signaling $[17,18]$, and flowering time [19]. Plant defense signaling is also regulated by miRNAs in response to different abiotic stresses [20,21] including heat, cold, drought [22,23], and UV-B 
radiation [24]. For example, A. thaliana miR399 (AthmiR399), induced during phosphate starvation, targets the ubiquitin-conjugating E2 enzyme involved in phosphate uptake from the soil [25]. Under drought stress, Ath-miR159 regulates MYB33 and MYB101 transcription factors, which activate abscisic acid responses during seed germination [26]. Ath-miR398 regulates $\mathrm{Cu} / \mathrm{Zn}$-superoxide dismutase genes, which detoxify superoxide radicals [27]. A recent study reported that several miRNAs are induced upon mechanical wounding in tobacco leaves and roots [28].

Plant miRNAs are also involved in biotic interactions. Ath-miR393 is induced by flagellin-derived PAMP peptide 22, and targets the F-box protein and transport inhibitor response 1 , which plays a key role in antibacterial responses [29]. Ath-miR160, Ath-miR167, and AthmiR825 are induced in response to infection by Pst DC3000 hrcC [20], and A. thaliana and Nicotiana tabacum plants infected by TYMVp69 virus accumulate high levels of miR156, miR160, and miR164 [30,31]. Plant miRNAs are also involved in beneficial interactions with bacteria: miR482, miR1512, and miR1515 play a role during rhizobial infection in Glycine max nodulation with Bradyrhizobium japonicum [32]. However, little is known about the role of plant miRNAs in the response to insect herbivores.

The wild tobacco Nicotiana attenuata and its herbivore community have become an ecological model system for the study of plant-herbivore interactions. During attack by insect herbivores, $N$. attenuata rapidly induces jasmonate-mediated defense responses, which reconfigure primary and secondary metabolism [33,34]. Jasmonates comprise jasmonic acid (JA), its derivatives and conjugates; the jasmonates and in particular, the active hormone jasmonoyl-isoleucine (JA-Ile) regulate most defenses against chewing herbivores [35]. Fatty acid amino acid conjugates (FACs) in oral secretion (OS) from larvae of the specialist herbivore, Manduca sexta, trigger jasmonate-mediated direct and indirect defenses in $N$. attenuata, such as nicotine accumulation, proteinase inhibitor production, diterpene glycoside biosynthesis, and emission of green leaf volatiles [36-38]. Transgenic plants impaired in jasmonate biosynthesis or signaling show increased susceptibility to herbivory in both glasshouse and field studies [35,39-41].

OS-elicitation dramatically changes the smRNA population in $N$. attenuata [42], and two major components of the smRNA pathway, RdRs and DCL proteins, function in biotic and abiotic stress responses [42-45]. Silencing of $N$. attenuata RdR1, DCL3, and DCL4 results in impaired defense responses against $M$. sexta herbivory [42,44,45]. Silencing either NaRdR1 or NaDCL4 impairs jasmonic acid (JA) accumulation, and co-silencing NaDCL3 and NaDCL4 reduces JA levels, indicating that
RdR1/DCL4-mediated smRNAs are critical regulators of responses to insect herbivory.

To deepen our understanding of the roles that smRNAs play in plant-insect interactions, we identified primary miRNA $(M I R)$ transcripts and TAS transcripts encoding tasiRNAs in a transcriptome database of $N$. attenuata [46], and computationally analyzed secondary stem-and-loop structures of MIR transcripts. To understand the role of jasmonates in regulating miRNAs, we examined miRNA accumulation in jasmonate-deficient allene oxide cyclase (AOC) RNAi lines. The AOC protein provides a precursor for JA biosynthesis [47]. Expression analysis of miRNAs and tasiRNAs with their putative target genes provides evidence for a key role of plant smRNAs in the response to herbivory.

\section{Results and discussion}

\section{Identification of conserved miRNAs and their precursors} in N. attenuata

To identify conserved miRNAs in $N$. attenuata, we used a 454-transcriptome database of $N$. attenuata to conduct a BLAST search against conserved plant miRNAs in the miRBase (www.mirbase.org) (Figures 1A and 1B). This search identified 59 potential miRNAs distributed in 36 families (Table 1). We used the BLASTX algorithm against the NCBI protein database to check that the putative primary transcripts of miRNAs were noncoding. Web-based mFOLD software (http://mfold.rna. albany.edu/) was used to predict secondary stem-andloop structures. Of the identified miRNA-precursors, 52 had stem-and-loop structures (Figure $1 \mathrm{C}$ and Additional file 1), which were created with minimum free energies (MFE) ranging from $\Delta G=-97.5 \mathrm{kcal} \mathrm{mol}^{-1}$ to -33.3 $\mathrm{kcal} \mathrm{mol}^{-1}$ (Table 1) with an average MFE of $-62.1 \mathrm{kcal}$ $\mathrm{mol}^{-1}$. This average MFE is comparable to that found in A. thaliana $\left(-59.5 \mathrm{kcal} \mathrm{mol}^{-1}\right)$, higher than in the red alga Porphyra yezoensis $\left(-41.7 \mathrm{kcal} \mathrm{mol}^{-1}\right)$ and lower than in the monocots rice $\left(-71.0 \mathrm{kcal} \mathrm{mol}^{-1}\right)$ and wheat $\left(-72.4 \mathrm{kcal} \mathrm{mol}^{-1}\right) \quad[22,48]$. Only seven predicted miRNA-precursors transcripts did not form stemand-loop structures or were not stable (Table 1). We identified several $N$. attenuata (Nat) miRNA families (Nat-miR403, Nat-miR478, Nat-miR482, Nat-miR1128, NatmiR1133, Nat-miR1446, Nat-miR1863, Nat-miR2911, and Nat-miR5281) which were not reported in N. tabaccum $[28,49]$. Among these, Nat-miR478, Nat-miR482, NatmiR1128, Nat-miR1133, Nat-miR1446, Nat-miR1863, and Nat-miR5281are absent in A. thaliana but are close homologues to those in other plant species (Table 1).

Next, we designed probes to detect $N$. attenuata miRNAs on RNA blots (Additional file 2). We performed northern blot hybridization using $40 \mu \mathrm{g}$ of total RNA extracted from rosette leaves to detect selected miRNAs. Accumulation of miRNAs varied 


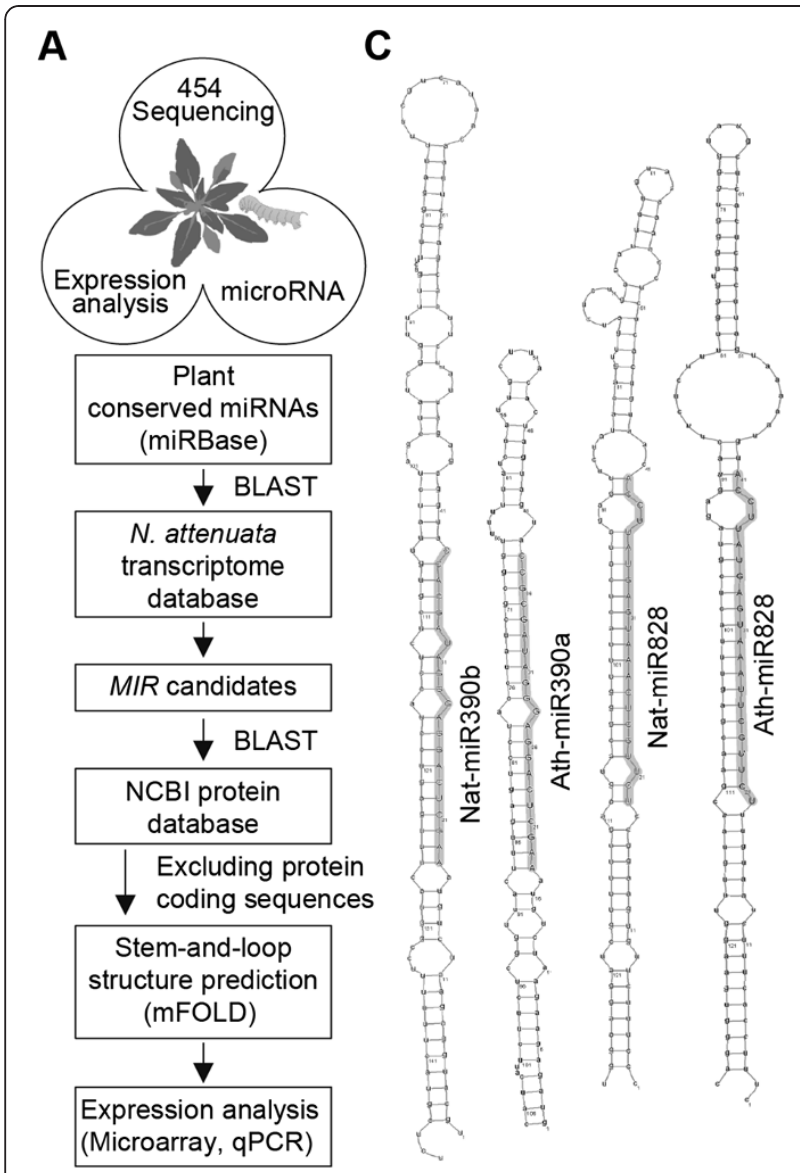

B

$$
\begin{aligned}
& \text { Nat-miR390 5' - AAGCUCAGGAGgGAUAGCACC - 3' } \\
& \text { Gma-miR390 5' - AAGCUCAGGAGGGAUAGCACC - 3' } \\
& \text { Ath-miR390 5' - AAGCUCAGGAGGGAUAGCGCC - 3' } \\
& \text { Nat-miR828 5' - UCUUGCUCAAAUGAGUAUUCCA - 3' }
\end{aligned}
$$

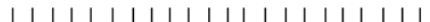

$$
\begin{aligned}
& \text { Vvi-miR828 5' - UCUUGCUCAAAUGAGUAUUCCA - 3' } \\
& \text { Ath-miR828 5' - UCUUGCUUAAAUGAGUAUUCCA - 3' }
\end{aligned}
$$

Figure 1 Identification and prediction of miRNAs in $\mathbf{N}$. attenuata. (A) A workflow depicting miRNA identification in N. attenuata. MIR, primary miRNA transcript. (B) Examples of conserved $N$. attenuata miRNAs with orthologs in plant species. Ath, Arabidopsis thaliana; Gma, Glycine max; Nat, Nicotiana attenuata; Vvi, Vitis vinifera. (C) Stem-and-loop structures of Nat-miR390 and Nat-miR828 precursors. Hairpin structures are compared to Arabidopsis miRNA orthologs based on their miRNAs sequence similarity. The miRNAs are highlighted in structures.

(Additional file 3). Accumulation of Nat-miR159, NatmiR171, Nat-miR172, and Nat-miR319 was high compared to Nat-miR157, Nat-miR393, Nat-miR396, and Nat-miR828 in leaves from rosette-stage plants. For further analyses of precursor and mature miRNA abundance, we used real-time quantitative PCR (qPCR) with specific primer sets (Additional file 4 and 5).

\section{Identification of conserved tasiRNAs in N. attenuata}

Four families of endogenous tasiRNAs (TAS1, TAS2, TAS3, and TAS4) identified in A. thaliana are regulated by miRNAs $[9,50]$. We found three TAS3 transcripts and one TAS4 transcript in N. attenuata (Figure 2), and constructed a phylogenetic tree with their homologs from different plant species to examine the evolutionary relationships of TAS3 expressed in dicotyledonous and monocotyledonous plant species [50,51]. Not surprisingly, NaTAS3 members were grouped amongst members of the dicotyledonous plant species (Figure 2A).

Because TAS3 and TAS4 transcripts contain the binding sites of miR390 and miR828, respectively [9,11,52], we blasted Nat-miR390 and Nat-miR828 against the TAS transcripts (Figure 2B), and compared the $N$. attenuata tasiRNAs with those in A. thaliana (Figure 2C). Sequence analysis indicated that binding sites of miRNA and tasiRNA sequences are highly conserved in $N$. attenuata and $A$. thaliana.

\section{Wound- and OS-inducible miRNAs}

Next, we examined the abundance of miRNAs and their target genes in leaves of rosette-stage WT plants changed after wounding and the application of diluted $M$. sexta OS (W+OS) or water to wounds (W+W, as a control for $\mathrm{W}$ $+\mathrm{OS}$ ) versus no treatment (control) (Figures 3 and 4). W + OS treatment faithfully mimics the majority of responses elicited by $M$. sexta feeding [36,38], but has the distinct advantage of allowing the time of elicitation to be precisely controlled (as opposed to the sporadic nature of M. sexta larval feeding behavior) and hence greatly increases the reproducibility of transcriptional analyses.

The abundance of mature Nat-miR159, Nat-miR160, NatmiR167, Nat-miR396, Nat-miR403, Nat-miR408, and NatmiR828 increased after both $\mathrm{W}+\mathrm{W}$ and $\mathrm{W}+\mathrm{OS}$ treatments, abundances of which did not differ (Figure 3A). We categorized these miRNAs wound-inducible miRNAs. Twelve conserved miRNA families (Nat-miR156, Nat-miR164, Nat-miR166, Nat-miR168, Nat-miR171, Nat-miR172, NatmiR319, Nat-miR390, Nat-miR393, Nat-miR394, NatmiR398, and Nat-miR1446) were significantly increased in $\mathrm{W}+\mathrm{OS}$ treated leaves compared to control and $\mathrm{W}+\mathrm{W}$ treated leaves (Figure 3B) and these were classified as OSinducible miRNAs.

It is well-established that miRNAs and tasiRNAs bind to their target mRNAs by perfect or imperfect complementarity [5]. Such complementarity permits the identification of miRNA targets in databases. We blasted miRNA sequences against an in-house transriptomic database using BLASTN algorithms with default parameters allowing 1-4 mistmatches. We identified 111 potential targets, inluding targets of tasiRNAs (Additional file 6).

Ath-miR164 is known to negatively regulate ORE1/ NAC2 transcription factors which are involved in age- 
Table 1 Identification and prediction of miRNAs in N. attenuata

\begin{tabular}{|c|c|c|c|c|c|c|}
\hline $\begin{array}{l}\text { Nat-MIR family } \\
\text { members }\end{array}$ & MFE & miRNA & Length (nt) & $\frac{\text { GC content }}{(\%)}$ & Hit in miRBase & E-value \\
\hline Nat-miR156a & -55.1 & ugacagaagagagugagcaca & 21 & 47.6 & bna-miR156 & 0.001 \\
\hline Nat-miR156b & -76.5 & ugacagaagagagugagcaca & 21 & 47.6 & bna-miR156 & 0.001 \\
\hline Nat-miR157 & -43.9 & uugacagaagauagagagcac & 21 & 42.9 & ath-miR157 & 0.001 \\
\hline Nat-miR159a & -62.3 & uuuggauugaagggagcucua & 21 & 42.9 & ath-miR159 & 0.001 \\
\hline Nat-miR159b & -90.6 & uuuggauugaagggagcucua & 21 & 42.9 & ath-miR159 & 0.001 \\
\hline Nat-miR159c & -104.3 & uuuggauugaagggagcucua & 21 & 42.9 & ath-miR159 & 0.001 \\
\hline Nat-miR160a & -61.6 & ugccuggcucccuguaugcca & 21 & 61.9 & ath-miR160 & 0.001 \\
\hline Nat-miR160b & -63.3 & ugccuggcucccuguaugeca & 21 & 61.9 & ath-miR160 & 0.001 \\
\hline Nat-miR162 & -43.9 & uggaggcagcgguucaucgauc & 22 & 54.5 & csi-miR162 & 0.0001 \\
\hline Nat-miR164 & -74.2 & uggagaagcagggcacgugca & 21 & 61.9 & ath-miR164 & 0.001 \\
\hline Nat-miR166a & -59.8 & ucggaccaggcuucauucccc & 21 & 61.9 & ath-miR166 & 0.001 \\
\hline Nat-miR166b & -51 & ucggaccaggcuucauucccc & 21 & 61.9 & ath-miR166 & 0.001 \\
\hline Nat-miR166c & -71.8 & ucggaccaggcuucauucccc & 21 & 61.9 & ath-miR166 & 0.001 \\
\hline Nat-miR167a & -42 & ugaagcugccagcaugaucua & 21 & 47.6 & ath-miR167 & 0.001 \\
\hline Nat-miR167b & -47 & ugaagcugccagcaugaucua & 21 & 47.6 & ath-miR167 & 0.001 \\
\hline Nat-miR168 & -78.3 & cccgccuugcaucaacugaau & 21 & 52.4 & aly-miR168 & 0.001 \\
\hline Nat-miR169a & -86.1 & cagccaaggaugacuugccga & 21 & 57.1 & ath-miR169 & 0.001 \\
\hline Nat-miR169b & -70.5 & uagccaaggaugacuugccugc & 22 & 50.0 & bna-miR169 & 0.001 \\
\hline Nat-miR171a & -70.1 & ugauugagecgcgucaauauc & 21 & 47.1 & vvi-miR171 & 0.001 \\
\hline Nat-miR171b & -61.4 & ugauugagccgcgucaauauc & 21 & 47.1 & vvi-miR171 & 0.001 \\
\hline Nat-miR171c & -44.9 & ugauugagecgcgucaauauc & 21 & 47.1 & vvi-miR171 & 0.001 \\
\hline Nat-miR171d & -60.4 & ugauugagecgcgucaauauc & 21 & 47.1 & vvi-miR171 & 0.001 \\
\hline Nat-miR172a & -53.4 & ugagaaucuugaugaugcugcau & 23 & 39.1 & vvi-miR172 & 0.001 \\
\hline Nat-miR172b & -49.5 & ugagaaucuugaugaugcugcau & 23 & 39.1 & vvi-miR172 & 0.001 \\
\hline Nat-miR172C & -61.7 & ugagaaucuugaugaugcugcau & 23 & 39.1 & vvi-miR172 & 0.001 \\
\hline Nat-miR172d & -35.2 & ugagaaucuugaugaugcugcau & 23 & 39.1 & vvi-miR172 & 0.001 \\
\hline Nat-miR319a & -97.5 & unggacugaagggagcuсcсu & 21 & 57.1 & ath-miR319 & 0.001 \\
\hline Nat-miR319b & -89.7 & uuggacugaagggagcucccu & 21 & 57.1 & ath-miR319 & 0.001 \\
\hline Nat-miR319c & -95.4 & uuggacugaagggagcuсcсu & 21 & 57.1 & ath-miR319 & 0.001 \\
\hline Nat-miR390a & -67.6 & aagcucaggagggauagcacc & 21 & 57.1 & gma-miR390 & 0.001 \\
\hline Nat-miR390b & -58.1 & aagcucaggagggauagcacc & 21 & 57.1 & gma-miR390 & 0.001 \\
\hline Nat-miR393a & -41.7 & uccaaagggaucgcauugaucc & 21 & 45.5 & ath-miR393 & 0.0004 \\
\hline Nat-miR393b & -54.4 & uccaaagggaucgcauugaucc & 21 & 45.5 & ath-miR393 & 0.0004 \\
\hline Nat-miR394a & -41.7 & unggcauucuguccaccuccau & 22 & 50.0 & vvi-miR394 & 0.0004 \\
\hline Nat-miR394b & -82.3 & unggcauucuguccaccuccau & 22 & 50.0 & vvi-miR394 & 0.0004 \\
\hline Nat-miR396 & -56.4 & unccacagcuuucuugaacug & 22 & 42.9 & ath-miR396 & 0.001 \\
\hline Nat-miR397 & -49.9 & ucauugagugcagcguugaug & 22 & 47.6 & ath-miR397 & 0.001 \\
\hline Nat-miR398 & -69.1 & uguguucucaggucaccccuu & 21 & 52.4 & ath-miR398 & 0.001 \\
\hline Nat-miR399 & - & ugccaaagaagauuugecccgu & 21 & 52.4 & ptc-miR399 & 0.001 \\
\hline Nat-miR403 & -38.2 & uuagauucacgcacaaacucg & 21 & 42.9 & ath-miR403 & 0.001 \\
\hline Nat-miR408 & -47.6 & augcacugccucuucccuggc & 21 & 61.9 & ath-miR408 & 0.001 \\
\hline Nat-miR413 & - & cuaguuucucuuguucugcuu & 21 & 38.1 & ath-miR413 & 0.015 \\
\hline Nat-miR414 & - & uccucuucaucaucaucuuc & 21 & 40.0 & ath-miR414 & 0.074 \\
\hline
\end{tabular}


Table 1 Identification and prediction of miRNAs in N. attenuata (Continued)

\begin{tabular}{|c|c|c|c|c|c|c|}
\hline Nat-miR477 & -54.8 & acucucccucaagggcuucug & 21 & 57.1 & aqc-miR477 & 0.001 \\
\hline Nat-miR478 & -33.3 & ugacaugucuuauauuuuuag & 20 & 23.8 & ptc-miR478 & 0.005 \\
\hline Nat-miR482 & -53.1 & uuuccaauuccacccauuccua & 21 & 40.9 & sly-miR482 & 0.0004 \\
\hline Nat-miR828 & -46.7 & ucuugcucaaaugaguauucca & 21 & 36.4 & vvi-miR828 & 0.0004 \\
\hline Nat-miR845a & - & ugcucugauaccaaauugaug & 22 & 38.1 & ath-miR845 & 0.003 \\
\hline Nat-miR845b & - & uggcucugauaccaauugau & 22 & 40.0 & vvi-miR845 & 0.004 \\
\hline Nat-miR1128 & -85.4 & uacuacucccuccguuucaa & 20 & 45.0 & ssp-miR1128 & 0.081 \\
\hline Nat-miR1133 & -88.1 & cauauacucccuccgucccugaaa & 21 & 50.0 & tae-miR1133 & 0.017 \\
\hline Nat-miR1446 & -48.7 & uucugaacucucucccucaa & 20 & 45.0 & ptc-miR1446 & 0.003 \\
\hline Nat-miR1863a & - & gcucugauaccauguuaacu & 24 & 40.0 & osa-miR1863b & 0.008 \\
\hline Nat-miR1863b & - & gacucugauaccauguuaaaauag & 20 & 28.0 & osa-miR1863 & 0.02 \\
\hline Nat-miR2911 & -67.3 & ggccgggggacggacuggga & 20 & 80.0 & peu-miR2911 & 0.014 \\
\hline Nat-miR5281a & -51.9 & cauauaaauugaaacggagggag & 23 & 39.1 & mtr-miR5281b & 0.13 \\
\hline Nat-miR5281b & -69.9 & cauauaaauugaaacggagggag & 23 & 39.1 & mtr-miR5281b & 0.13 \\
\hline Nat-miR5281c & -34.2 & cauauaaauugaaacggagggag & 23 & 39.1 & mtr-miR5281b & 0.13 \\
\hline
\end{tabular}

dependent senescence [53] and mutations in ORE1/ NAC2 delays chlorophyll loss in old leaves of $A$. thaliana [53]. We found a NAC transcription factor containing a Nat-miR164 binding site in $N$. attenuata (Figure 4). The NAC transcripts were significantly reduced in $\mathrm{W}+\mathrm{W}$ and $\mathrm{W}+\mathrm{OS}$ elicited leaves (Figure 4), which correlated with elevated Nat-miR164 levels after OS-elicitation (Figure 3B). This result may explain how $M$. sexta attack delays senescence in $N$. attenuata [54].

In addition, ARF6 and ARF8 were putative targets of wound-inducible Nat-miR167, and their transcripts decreased concurrently with the accumulation of NatmiR167 (Figure 4). Potential targets of OS-inducible Nat-miR171, GRAS domain transcription factors, were down-regulated in OS-elicited plants (Figure 4). Transcripts of several OS-inducible Nat-miR156 targets annotated as SQUAMOSA promoter binding proteins (SBPs) were significantly down-regulated after $\mathrm{W}+\mathrm{OS}$ treatment. Abundance of Nat-miR172 increased significantly in $\mathrm{W}+\mathrm{OS}$ treated leaves (Figure 3B) and consistently, transcripts of its putative targets, AP2-like proteins, were significantly down-regulated in $\mathrm{W}+\mathrm{W}$ and W+OS treated leaves (Figure 4).

The most significant change in miRNA transcripts after W+OS treatment was for Nat-miR390, which showed a 75-fold increase compared to control leaves and a 30-fold increase compared to $\mathrm{W}+\mathrm{W}$ treated leaves (Figure 3B). Ath-miR390 cleaves TAS transcripts, resulting in production of tasiRNA3 mediated by the RdR6/ DCL4 complex [11,52]. TasiRNA3 controls the transcription of auxin response factors (ARFs) 2, 3, and 4, which regulate leaf morphology and lateral root growth in $A$. thaliana $[11,16,52]$. Overexpression of TAS3 leads to an increased number of lateral roots, and the knockout mutant shows impaired lateral root growth [11]. Abundance of mature Nat-tasiRNA3 in W+OS treated leaves was increased (Figure 5A) and Nat-tasiRNA3 was regulated in a JA-independent manner (Figure 5B). The targets of tasiRNA3, transcripts homologous to $A$. thaliana $A R F 2,3$ and 4 were significantly reduced after W +OS elicitation (Figure 4), suggesting that Nat-miR390 could affect the architecture of roots and thereby regulate the production of nicotine, which is synthesized in the roots, or promote tolerance of herbivory by increasing mineral uptake or sugar storage in roots. Our previous study shows that silencing NaDCL4, which in turn reduces the accumulation of tasiRNA3, dramatically impairs root growth and nicotine accumulation in $N$. attenuata [45]. We validated the microarray data by qPCR analysis (Additional file 7), and the result showed similar expression patterns (Additional file 8).

\section{JA-dependent miRNAs}

The key role of jasmonates in mediating responses to herbivory is well established and it was not surprising to find that the levels of jasmonates dramatically increased in $\mathrm{W}+\mathrm{OS}$ treated leaves compared to $\mathrm{W}+\mathrm{W}$ treatment (Additional file 9) [37]. Silencing of NaAOC reduces JA accumulation in W+OS treated leaves by $90-100 \%$ (Additional file 9) and is known to silence the production of JA-mediated defenses [47]. OS-elicitation of this genotype allowed us to understand which miRNAs are regulated by jasmonates.

Abundance of 10 miRNAs (Nat-miR159, Nat-miR160, Nat-miR164, Nat-miR166, Nat-miR168, Nat-miR171, Nat-miR172, Nat-miR393, Nat-miR403, and Nat- 


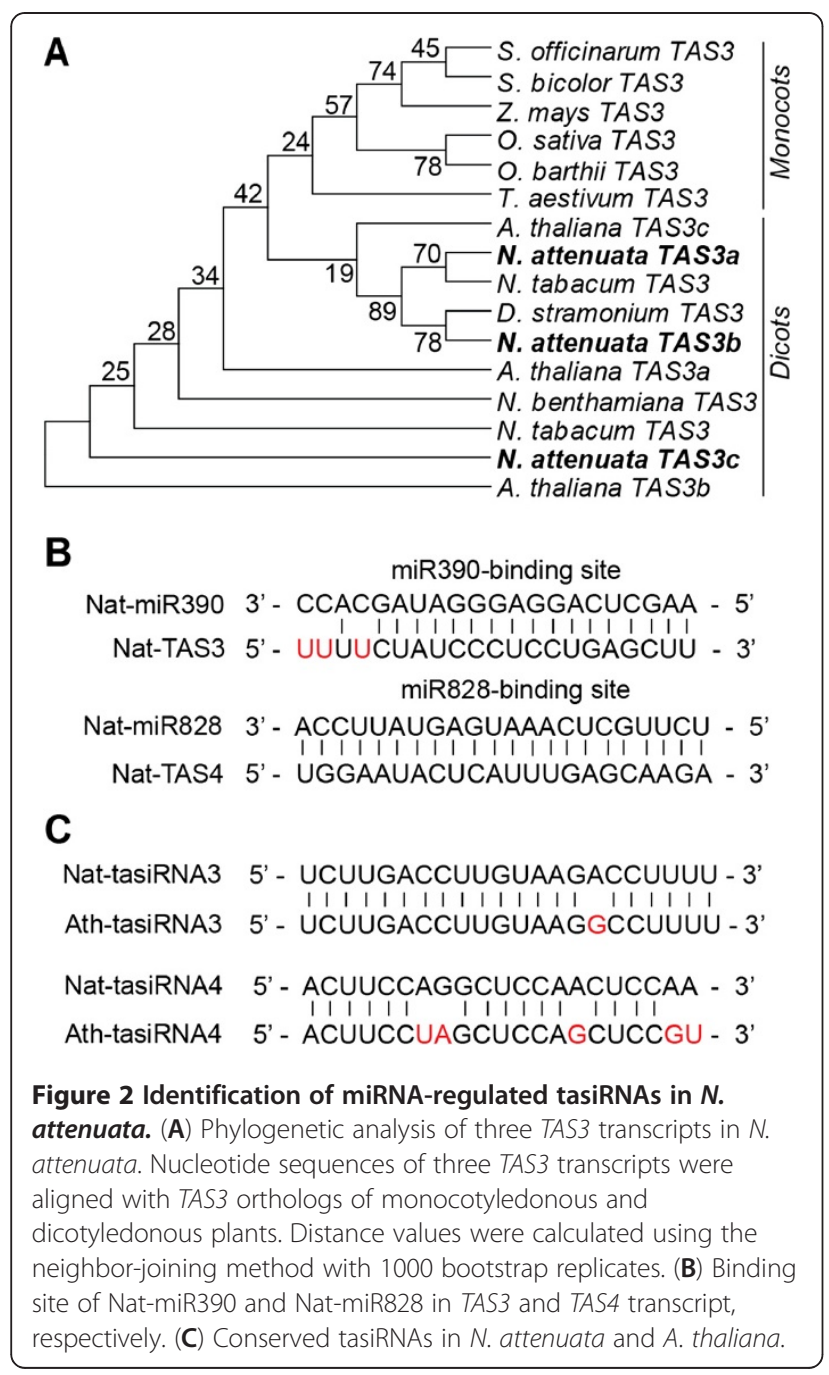

miR408) increased after $\mathrm{W}+\mathrm{W}$ and $\mathrm{W}+\mathrm{OS}$ treatments, but did not differ between WT and ir-aoc plants (Figure 6A). We considered these miRNAs as JAindependent miRNAs. The abundance of Nat-miR156, Nat-miR167, Nat-miR390, Nat-miR396, Nat-miR398, and Nat-miR1446 were significantly higher in both control and $\mathrm{W}+\mathrm{OS}$ elicited leaves of ir-aoc than of WT plants (Figure 6B), indicating that jasmonates or JAsignaling negatively influences levels of these miRNAs, regardless of treatment. Nat-miR390 abundance was higher in ir-aoc only after W+OS treatment (Figure 6B). While abundance of Nat-miR319 and Nat-miR394 were induced by $\mathrm{W}+\mathrm{OS}$ treatment in WT (Figure 3B), they were not induced by $\mathrm{W}+\mathrm{OS}$ treatment in ir-aoc plants (Figure 6C). We considered these as OS-inducible JAdependent miRNAs. Wound-inducible Nat-miR828 was induced in both $\mathrm{W}+\mathrm{W}$ and $\mathrm{W}+\mathrm{OS}$ treatments in WT (Figure 3A), but their levels were not dramatically induced in elicited JA-deficient ir-aoc plant compared to WT (Figure 6C).
One main target of miR319 in A. thaliana is TCP (TEOSINTEBRANCHED/CYCLOIDEA/PCF) transcription factors, which positively regulates jasmonate biosynthesis [18]. The TCP4 protein can bind a promoter of LIPOXYGENASE2 (LOX2) in A. thaliana [18]. We do not yet know the orthologs of $A$. thaliana TCPs in $N$. attenuata, but the timing of Nat-miR319 induction was similar with that of $\mathrm{W}+\mathrm{OS}$ elicited JA accumulation and the induction of NaLOX3, which is the functional homo$\log$ of $A$. thaliana LOX2 $[40,55]$. W+OS treatment amplifies the wound-induced JA accumulation (Additional file 9) [36,38] and does the same for Nat-miR319 (Figure 3B). This suggests that Nat-miR319 could play a role in the fine-tuning regulation of jasmonate biosynthesis [18] during herbivory.

Nat-miR828 and Nat-tasiRNA4 were increased by W $+\mathrm{W}$ and $\mathrm{W}+\mathrm{OS}$ treatments in WT (Figure 3A), but not in ir-aoc plants (Figures 5 and 6C). Levels of NatmiR828 dramatically decreased in ir-aoc plants compared to WT and its primary transcript as well (Figure 6C and Additional file 10). One target of miR828 is a TAS4 transcript, which encodes tasiRNA4 processed by RdR6/DCL4 proteins $[9,56]$. TasiRNA4 targets several MYB transcription factors which regulate phenylpropanoid biosynthesis $[9,56]$. Several phenylpropanoid defense metabolites increase in $N$. attenuata during herbivory and silencing of DCL4 alters the accumulation of dicaffeoyl spermidine and caffeoylputrescine in W+OSelicited leaves [45]. A jasmonate-inducible MYB transcription factor, NaMYB8, is involved in plant defense against herbivory and phenylpropanoid biosynthesis $[57,58]$. Although we were not able to find a MYB transcription factor containing a Nat-tasiRNA4 binding site in our current cDNA library, sequence conservation of miR828 and tasiRNA4 in A. thaliana and N. attenuata will guide the identification of targets which regulate secondary metabolite production.

\section{Temporal effect of single elicitation on miRNAs and their targets accumulation}

To examine the kinetics of miRNAs in response to insect herbivory, we measured the accumulation of miRNAs and their targets at $1 \mathrm{~h}$ and $5 \mathrm{~h}$ after treatments. Most OS-induced miRNAs increased in abundance within $1 \mathrm{~h}$ after OS-elicitation and continue to increase until $5 \mathrm{~h}$, except miR166 and miR390 (Additional file 11). The miRNA390 increased at $1 \mathrm{~h}$ but decreased again by $5 \mathrm{~h}$ post-elicitation (Additional file 11). OSinducible miRNAs were also induced by wounding but the increase after wounding was less (miR164, miR166, miR172, miR319, and miR398) or slower (miR168, miR390, and miR393) than that after OS-elicitation. Interestingly, most of the miRNA and tasiRNA target genes were reduced at $1 \mathrm{~h}$ after OS-elicitation but return 


\section{$\square \mathrm{CTRL} \square \mathrm{W}+\mathrm{W} \square \mathrm{W}+\mathrm{OS}$}

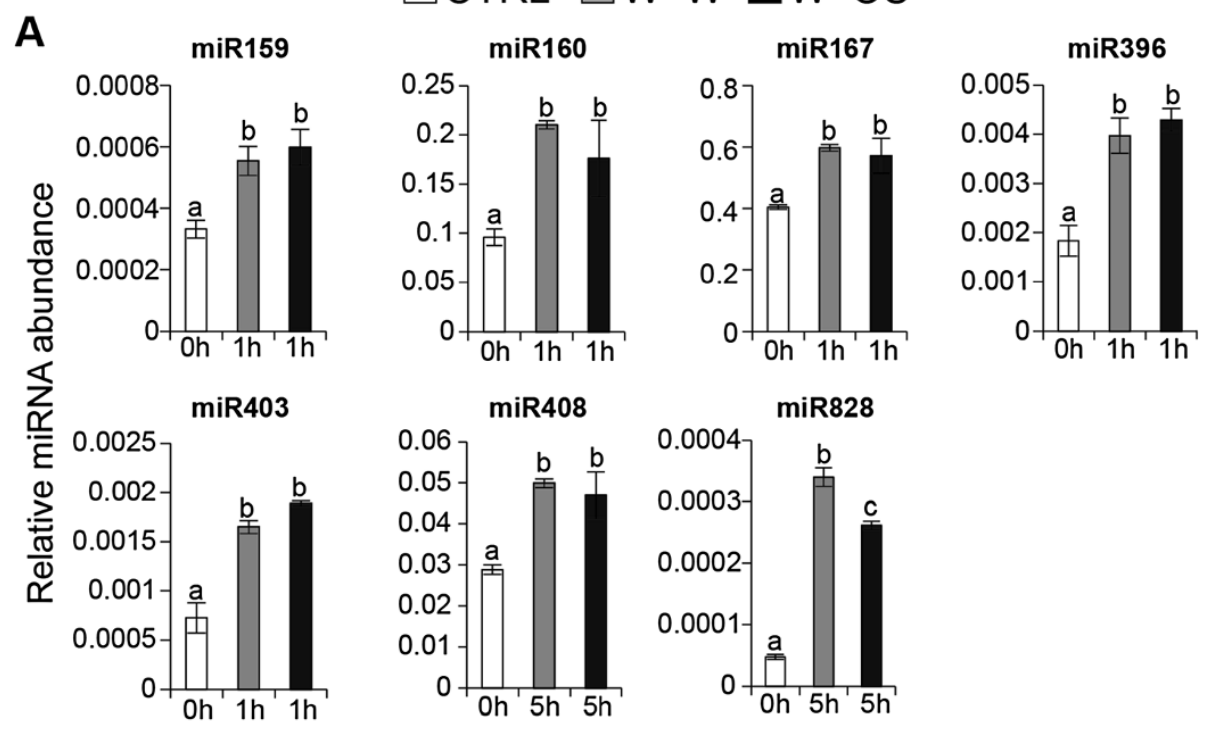

B
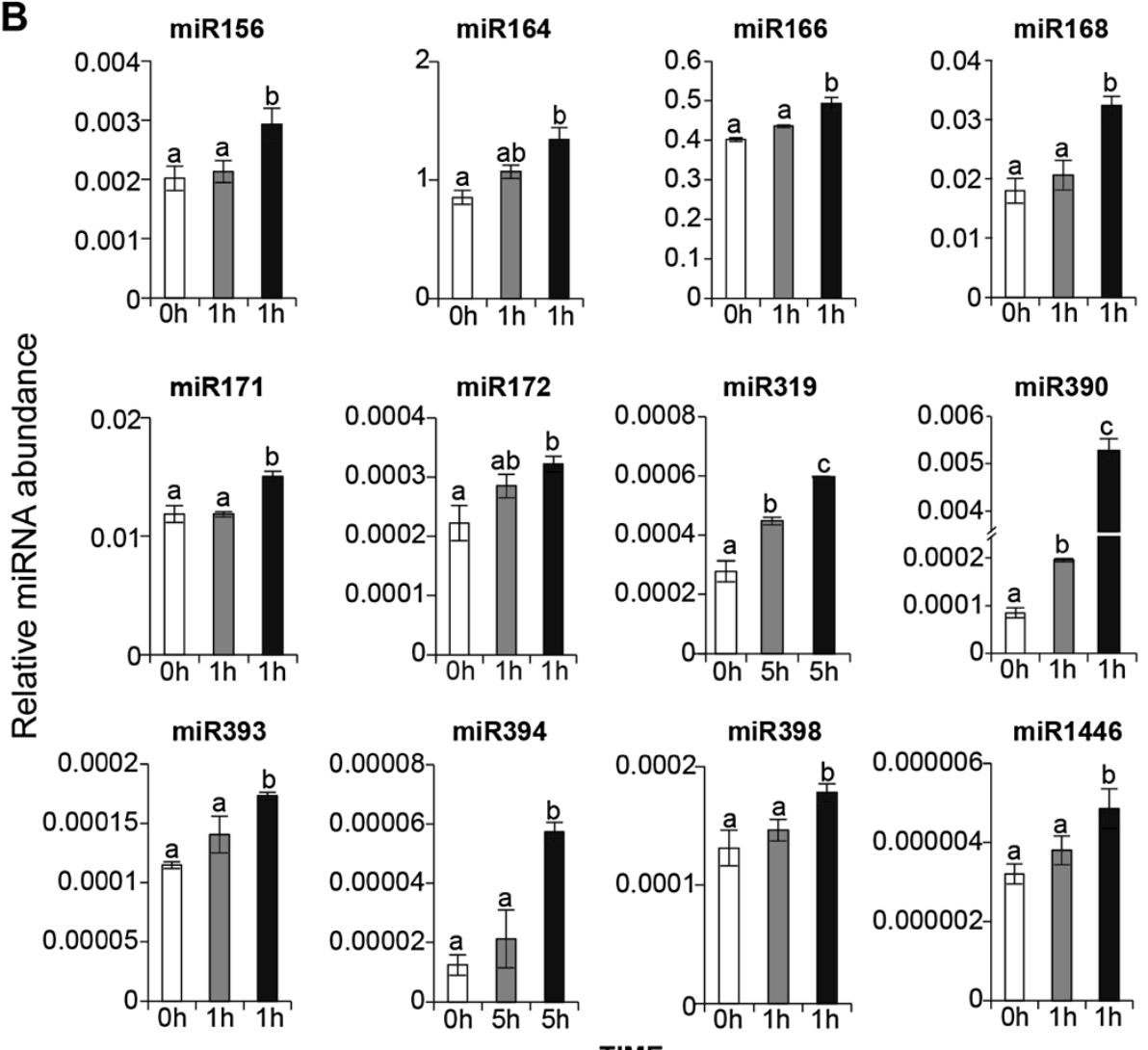

TIME

Figure 3 Wound- and OS-inducible miRNAs. (A) Wound-inducible miRNAs. (B) OS-inducible miRNAs. Effects of W+W and W+OS on relative transcript abundance of N. attenuata miRNAs. For each sample, one leaf on the rosette of a 32-day-old WT plant was left untreated. (control, CTRL) or treated with wounding plus water $(\mathrm{W}+\mathrm{W})$ or wounding plus oral secretions of the larvae of the specialist $M$. sexta (W+OS) and harvested $1 \mathrm{~h}$ or $5 \mathrm{~h}$ post treatment. Shown are mean $( \pm$ SE) levels of three replicates. Letters indicate significant differences $(P<0.05)$ in Fisher's PLSD tests following an ANOVA. 

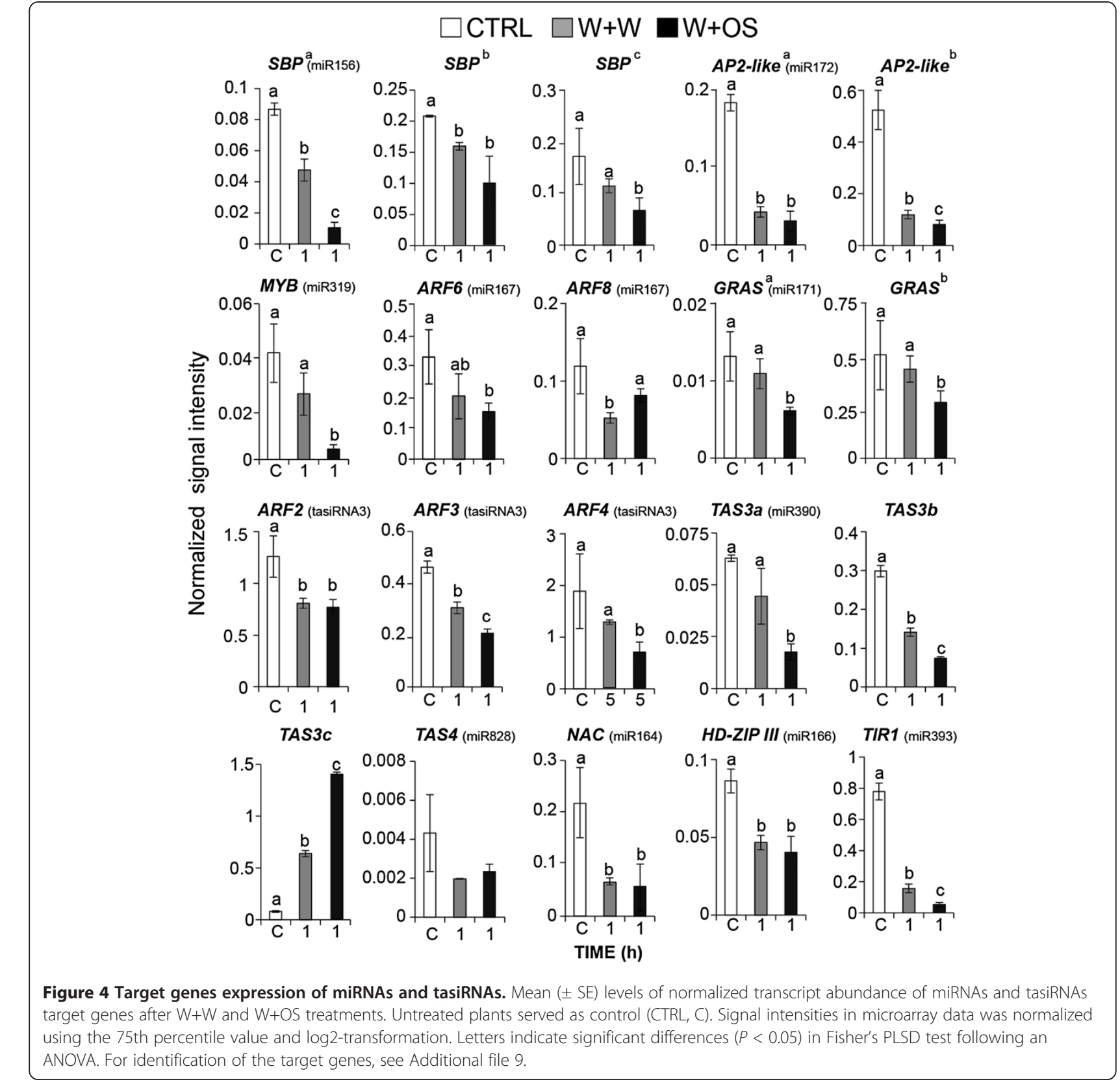

$\mathrm{W}+\mathrm{OS}$

to control levels (SBPa, ARF2, ARF3, TAS3a, TAS3b, HD-ZIPIII, TIR1) or even increased to levels greater than prior to elicitation (MYB, ARF6, ARF8), suggesting a complex relationship between the regulator mature miRNAs and their target genes.

\section{Comparison between primary and mature miRNA transcript accumulation}

We checked the accumulation of MIR transcripts using previously reported microarray data [46] (Additional file 12) and confirmed these responses by qPCR (Additional file 10). Accumulation of MIR156, MIR159, MIR164, MIR166, MIR167, MIR168, MIR172, MIR393, MIR396,
MIR398, MIR403, and MIR1446 was not correlated to the abundance of their mature miRNAs. Changes in transcript levels of MIR160, MIR171, MIR319, MIR390, MIR408, and MIR828 reflected the transcript accumulation of their mature miRNAs. Generally, a weak relationship between $M I R$ and mature miRNA accumulation has been reported [59]. Our data also suggests that the processing of $M I R$ transcripts is more important than the transcription of MIR genes in herbivory-induced miRNA regulation.

\section{Conclusion}

In order to build a database of plant miRNAs functionally involved in plant-insect interactions, we investigated the 


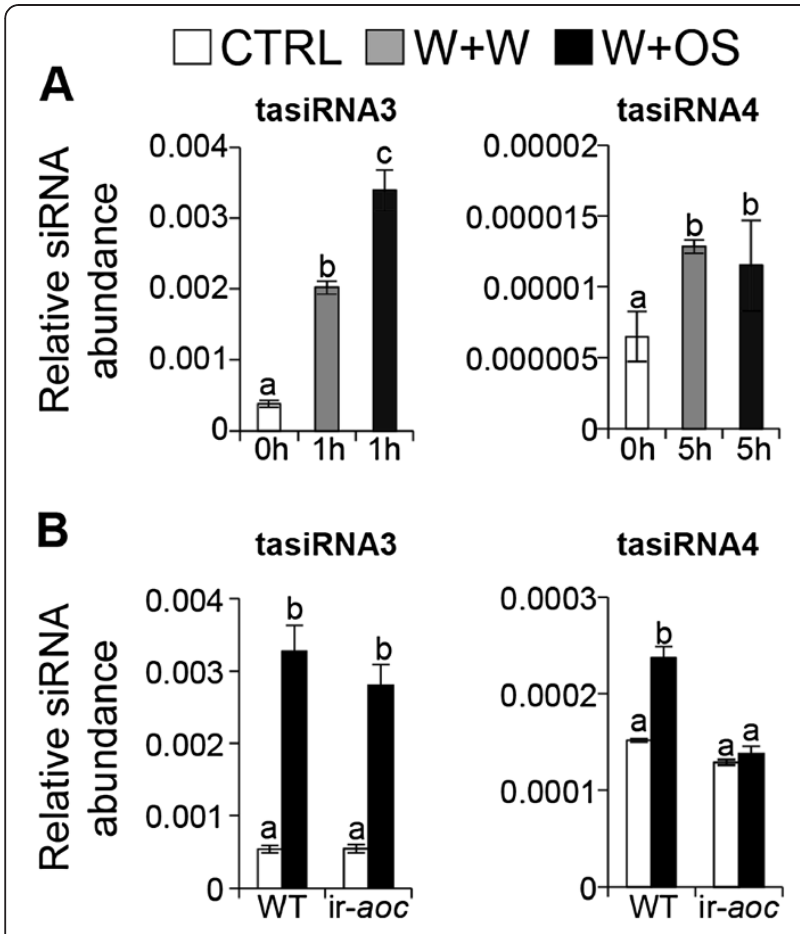

Figure 5 Accumulation of tasiRNAs in response to OS-elicitation. (A) Abundance of tasiRNA3, which is a target of the OS-inducible NatmiR390, significantly increased in OS-elicited plant compared to control (CTRL) and W+W treatment. Abundance of tasiRNA4, which is regulated by the wound-inducible Nat-miR828, was significantly increased by wounding. (B) Accumulation of JA-independent tasiRNA3 and JAdependent tasiRNA4 at $1 \mathrm{~h}$ and $5 \mathrm{~h}$ after OS-elicitation, respectively. For each sample, one leaf on the rosette of a 32-day-old plant was treated with wounding plus oral secretions of the larvae of the specialist herbivore $M$. sexta $(\mathrm{W}+\mathrm{OS})$ and harvested $1 \mathrm{~h}$ or $5 \mathrm{~h}$ post treatment. Untreated plants served as control. Shown are mean ( \pm SE) levels of three replicates. Letters indicate significant differences $(P<0.05)$ in Fisher's PLSD test following an ANOVA.

accumulations of miRNAs and their targets in N. attenuata after OS-elicitation. We classified W+OS-induced miRNAs and tasiRNAs into four groups: JA-dependent or -independent wound-inducible and OS-inducible miRNAs or tasiRNAs (Figure 7). Herbivore-attacked $N$. attenuata plants induce defense metabolites and tune their physiology to tolerate insect attack. This study shows that $\mathrm{W}+\mathrm{OS}$ elicitation, a rigorous means of mimicking herbivore attack, rapidly changed the expression of miRNAs involved in flowering time, root morphology, senescence, hormone regulation, and metabolite synthesis. In future work, we will experimentally characterize the function of these $\mathrm{W}+\mathrm{OS}$ regulated JA-dependent and JAindependent miRNAs and their targets.

\section{Methods}

Plant material and growth conditions

WT and JA-deficient ir-aoc RNAi lines of the 30th inbred generation of the N. attenuata (Torrey ex. Watson) (originally collected in Southwestern Utah, USA) were used for the experiments. Seeds were germinated on MS basal medium supplemented with vitamin B5 (GB5, Duchefa, http://www.duchefa.com). For treatment, the leaf at the +1 node of the rosette (youngest fullyexpanded leaf) was wounded with a fabric pattern wheel, and either $20 \mathrm{uL}$ MilliQ water $(\mathrm{W}+\mathrm{W})$ or $20 \mu \mathrm{L}$ M. sexta OS (diluted 5X with sterile water) (W+OS) were applied. Leaves at the same position from untreated plants served as controls. Plants were grown in a glasshouse under 16/ $8 \mathrm{~h}$ (long day) $\left(26-28^{\circ} \mathrm{C}\right)$ supplemental light from Master Suns-U PIA Agro 400-W sodium lights.

\section{Identification of miRNAs and precursors, and prediction of miRNA targets}

To identify conserved miRNA and tasiRNA, we blasted all conserved plant miRNAs present in miRBase (www.mirbase.org) against $N$. attenuata's in house 454-transriptome database using default search parameters allowing 1 or 2 mismatches (Figure 1A). Hits of 20 to 24 nt sequences against non-coding transcripts with up to four nucleotide mismatches were selected as candidates to check for predicted miRNA secondary structure. The public web-based mFOLD server (http://mfold.rna.albany.edu/) was used to predict secondary stem-and-loop structures using default parameters, folding temperature $\left(37^{\circ} \mathrm{C}\right)$ and ionic conditions $(1 \mathrm{M} \mathrm{NaCl})$ with minimum free energy (MFE) formations (Table 1). Predicted miRNA-precursors were additionally depicted using RNAshapes (http://bibiserv. techfak.uni-bielefeld.de/rapidshapes) [60] to create stemand-loop structures (Additional file 1). For TAS3 identification we used the following TAS3 orthologs: Saccharum officinarum (EU327139), Sorghum bicolor (EU327137), Zea mays (EU327127, EU293143), Oryza barthii (GQ420228), Triticum aestivum (EU327134), A. thaliana TAS3a (AT3G17185), TAS3b (AT5G49615), TAS3c (At5g57735), Nicotiana tabacum (FJ804751, FJ804743), Nicotiana benthamiana (FJ804742), Datura stramonium (FJ804744). A neighbor-joining tree was built for TAS3 transcripts using MEGA4 with group evaluation and 1000 bootstrap replicates [61].

\section{RNA extraction and RNA blot hybridization}

RNA blot hybridization was performed as described by Molnar et al. (2007) [62]. Extracted total RNA was treated with DNase I (Fermentas; http://www.fermentas.com) according to the manufacturer's protocol. Enzymes were removed by phenol-chloroform extraction and total RNA was re-isolated by ethanol precipitation. A denaturing 15\% polyacrylamid gel containing $7 \mathrm{M}$ urea was prepared using the BIORAD Mini-Protean 3 Cell system (http://www.biorad.com). Forty $\mu \mathrm{g}$ of total RNA were denatured with an equal volume of $2 \mathrm{x}$ gel-loading dye (Fermentas, http:/ www.fermentas.de) at $65^{\circ} \mathrm{C}$ for 5 minutes. Denatured 

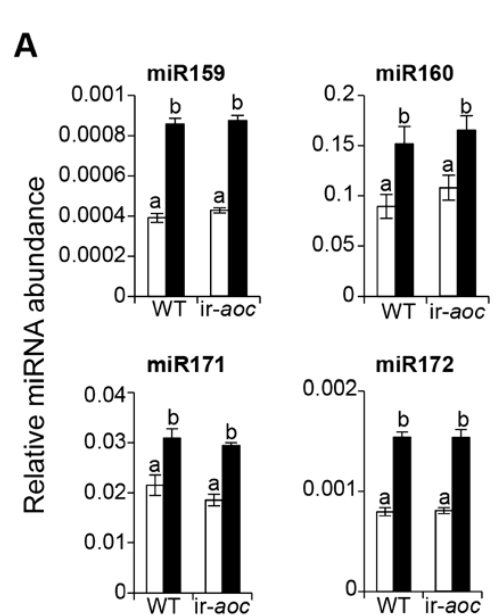

$\square \mathrm{CTRL} \quad \mathrm{W}+\mathrm{OS}$
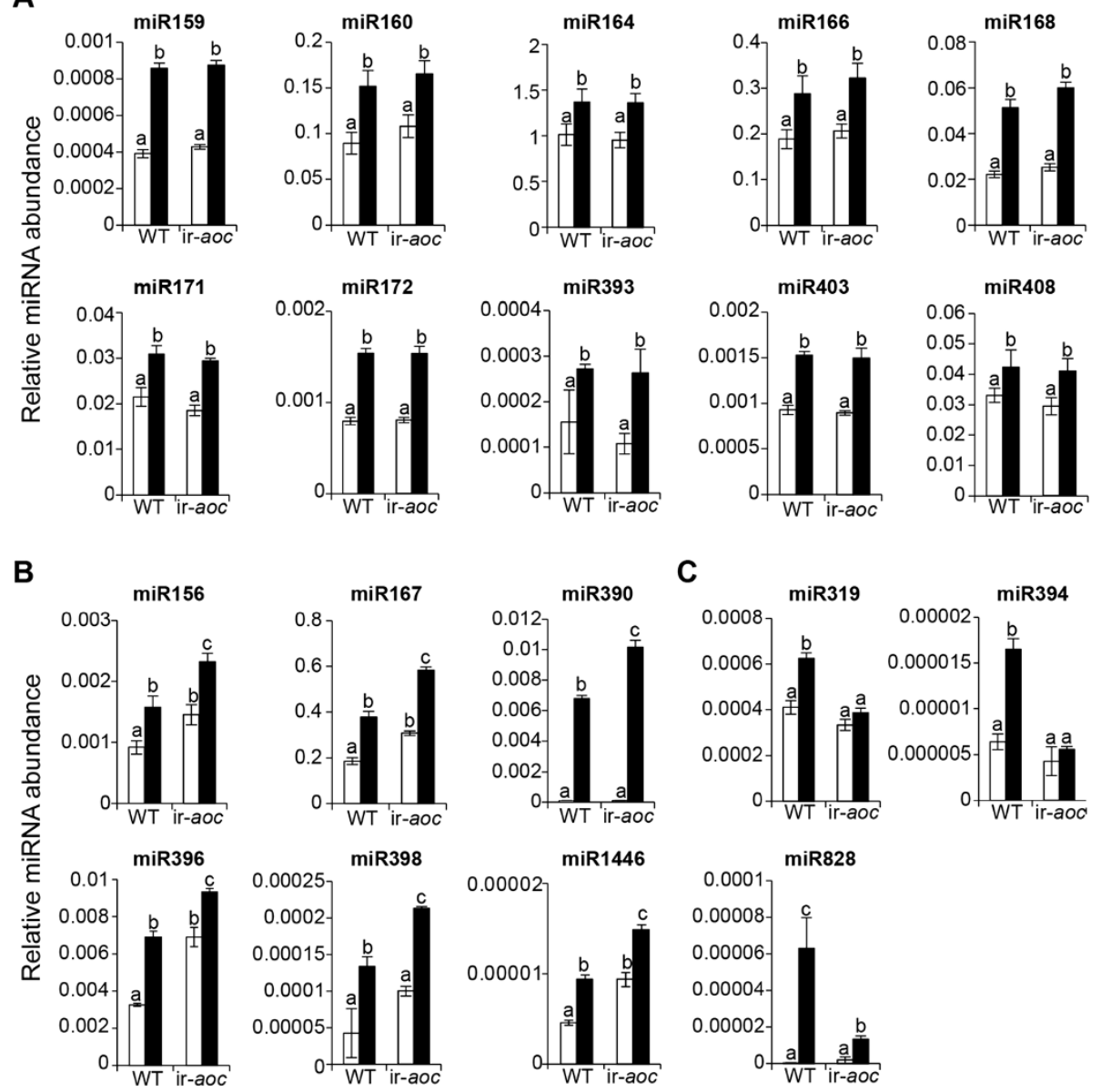

C
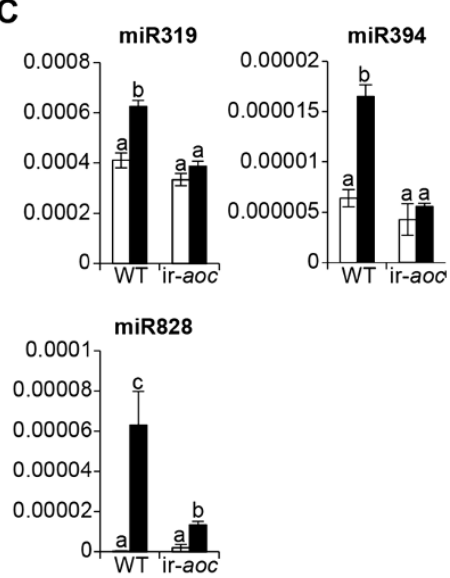

Figure $6 \mathrm{JA}$-independent and dependent miRNAs accumulation in response to OS-elicitation. (A) W+OS inducible and JA-independent miRNAs. (B) W+OS inducible miRNAs accumulated more in ir-aoc plant. (C) W+OS inducible and JA-dependent miRNAs. Shown are mean $( \pm$ SE) levels of miRNAs in control and elicited leaves at $1 \mathrm{~h}$ or $5 \mathrm{~h}$ after $\mathrm{W}+\mathrm{OS}$ treatment in wild-type (WT) and ir-aoc plants. For each sample, one leaf on the rosette of a 32-day-old WT and ir-aoc plant was left untreated (control, CTRL) or treated with wounding plus oral secretions of the larvae of the specialist herbivore $M$. sexta $(\mathrm{W}+\mathrm{OS})$ and harvested $1 \mathrm{~h}$ or $5 \mathrm{~h}$ post elicitation. Letters indicate significant differences $(P<0.05)$ in Fisher's PLSD tests following an ANOVA.

RNA samples were loaded into the prerun $15 \%$ denaturing polyacrylamid gel at $50 \mathrm{~V}$. A ZR small-RNA ${ }^{\mathrm{TM}}$ Ladder (Zymo Research, http://www.zymoresearch.com) was also loaded. The gel was run at $150 \mathrm{~V}$ until the bromphenol blue in the loading dye reached the bottom of the gel. Next, the portion of the gel containing small RNA (between the bromphenol blue and xylene cyanol) was cut out and blotted onto a nylon membrane (Nytran Supercharge; http://www.whatman.com) by capillary wet transfer overnight. Transferred RNA was crosslinked to the membrane with UV light at $1200 \mu \mathrm{J}$ (UV Stratalinker 2400, Stratagene; http://www.stratagene.com). The membrane was pre-hybridized in 5-10 mL of ULTRAhybOligo Hybridization Buffer (http://www.ambion.com) at $40^{\circ} \mathrm{C}$ for at least $30 \mathrm{~min}$. DNA oligo probes (Additional file 2) were labeled using ${ }^{32} \mathrm{P}$ with $\mathrm{U} 4$ polynucleotide kinase (Fermentas; http://www.fermentas.com). The reaction mix was incubated at $37^{\circ} \mathrm{C}$ for $1 \mathrm{~h}$. Unincorporated nucleotides were separated using a Microspin G-25 column according to the manufacturer's instructions (GE Healthcare, http:// www.gelifesciences.com). The labeled probe was mixed with the hybridization solution and hybridized to the membrane at $40^{\circ} \mathrm{C}$ overnight. The membrane was washed with a washing solution (2xSSC, $0.1 \% \mathrm{SDS})$ at $40^{\circ} \mathrm{C}$ for 10 minutes, wrapped with plastic saran wrap, and exposed to phospho-imaging plates.

\section{Quantitative real-time PCR (qPCR) and CDNA microarray}

One $\mu g$ of total RNA were reverse-transcribed using SuperScript ${ }^{\circledR}$ II reverse transcriptase following the manufacturer's protocol (Invitrogen; http://www.invitrogen. com). Twenty $\mu \mathrm{g}$ of cDNA was used to perform quantitative real-time PCR with SYBR Green using genespecific primers (Additional file 4 and 10) designed for 


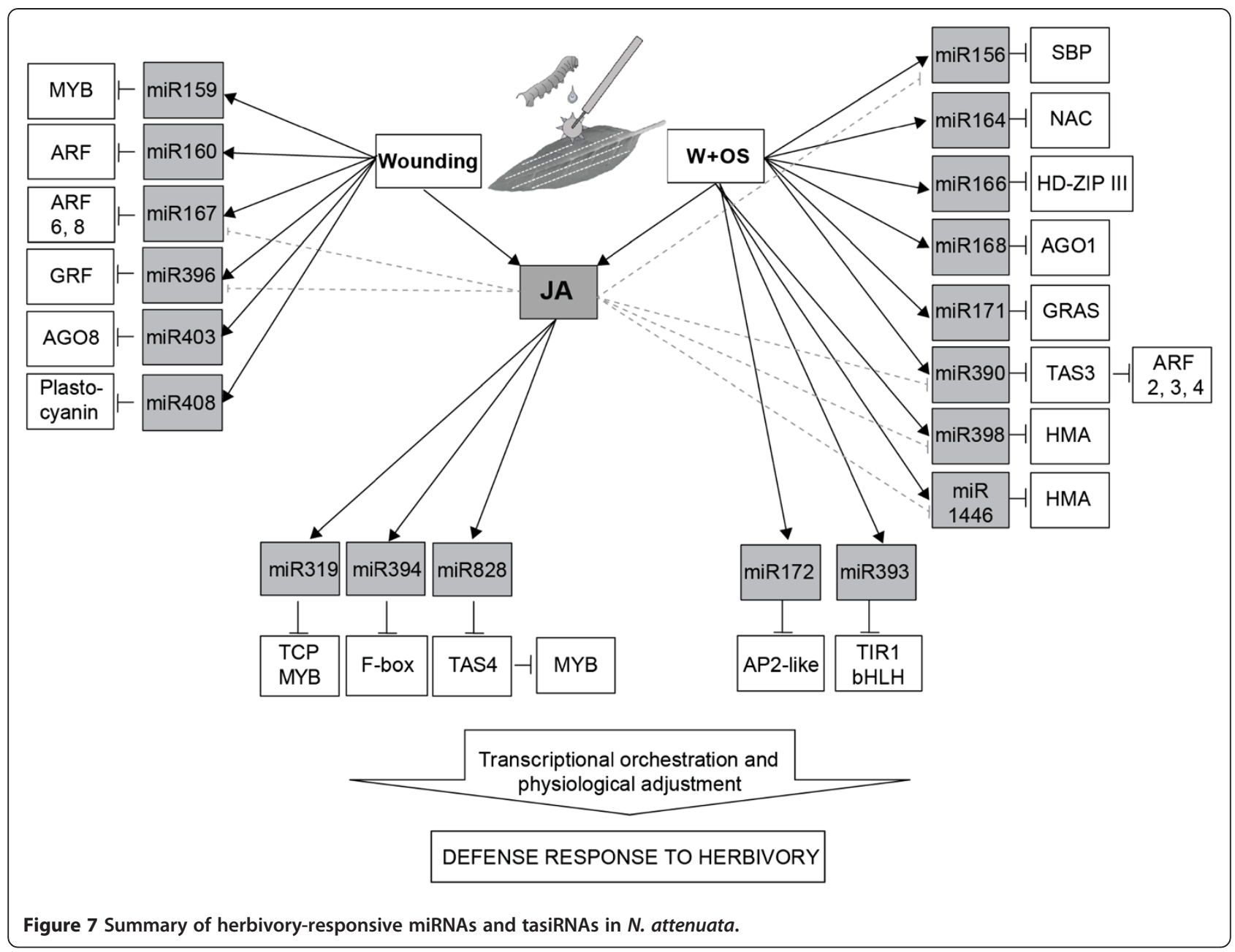

$M I R$ and target genes. Elongation factor $(\mathrm{NaEF})$ was used as reference house-keeping gene for analysis.

For reverse transcription of miRNA and tasiRNAs into cDNA, we used the miScript Reverse Transcription Kit (Qiagen; http://www.qiagen.com). $10 \mu \mathrm{g}$ of total RNA were used for qPCR (Additional file 5) with the miScript SYBR Green PCR Kit (Qiagen; http://www.qiagen.com) to quantify miRNAs and tasiRNAs. All qPCR data were analyzed using the $2 \Delta \Delta \mathrm{Ct}$ calculation method [63].

We used a cDNA microarray NCBI GEO database (Platform GPL13527, accession number GSE30287) [46]. For data analyses, raw data was normalized to the 75th percentile and log2-transformed. Comparisons with greater than a 2-fold change were tested by Fisher's PLSD test following an ANOVA.

\section{Phytohormone analysis}

JA and JA-Ile were co-extracted from leaf tissue as previously described [64]. One hundred to $150 \mathrm{mg}$ of lamina tissue from control and treated plants were used for phytohormone extraction with $1 \mathrm{ml}$ of ethyl acetate spiked with $200 \mathrm{ng}$ of $\mathrm{D}_{2}$-JA, and $40 \mathrm{ng}$ of ${ }^{13} \mathrm{C}_{6}$-JA-Ile as internal standards. Fifteen $\mu \mathrm{L}$ of the supernatant were analyzed on a Varian 1200 L Triple-Quadrapol-MS with a ProntoSIL column (C18; $5 \mu \mathrm{m}, 50 \times 2 \mathrm{~mm})$.

\section{Statistical analysis}

Data were calculated with the StatView Software using the one-way analysis of variance ANOVA (means were compared by the lowest standard deviation (LSD)) algorithm (SAS Institute Inc., Cary, NC, USA).

\section{Additional files}

Additional file 1: Stem-and-loop structures of identified miRNAs in N. attenuata.

Additional file 2: List of smRNA probes used for RNA blot hybridization.

Additional file 3: Accumulation of several miRNAs in rosette leaves of $\boldsymbol{N}$. attenuata. RNA blot hybridization performed to examine the accumulation of miRNAs in rosette leaves of $N$. attenuata. Ethidium bromide staining of rRNA is shown as a loading control.

Additional file 4: List of primers used for qPCR analysis of primary miRNAs. 
Additional file 5: List of smRNA-specific forward primers used for miScript qPCR.

Additional file 6: List of putative miRNA targets containing miRNA binding site.

\section{Additional file 7: Primer sequences of miRNA targets.}

Additional file 8: Transcript abundance of miRNA target genes.

Abundance of miRNA targets after $\mathrm{W}+\mathrm{W}$ and $\mathrm{W}+\mathrm{OS}$ treatments. For each sample, one leaf on the rosette of a 32-day-old WT plant was left untreated (control) or treated with wounding plus water $(W+W)$ or wounding plus OS (W+OS) and harvested $1 \mathrm{~h}$ or $5 \mathrm{~h}$ post treatment. Shown are mean $( \pm$ SE) levels of three replicates per line. Letters indicate significant differences $(P<0.05)$ in Fisher's PLSD tests following an ANOVA.

Additional file 9: JA and JA-lle levels were impaired in ir-aoc plants after elicitation. Accumulation of Jasmonic acid (JA) and jasmonoyl isoleucine (JA-\|e) increased in W+W and W+OS treated leaves and these accumulations were dramatically altered in ir-aoc plants. For each sample, one leaf on the rosette of a 32-day-old plant was treated and harvested 1 $\mathrm{h}$ or $5 \mathrm{~h}$ post treatment. Untreated plants served as control. Asterisks indicate significant differences $(* * *, P<0.001)$ in Fisher's PLSD tests following an ANOVA

\section{Additional file 10: qPCR data showing accumulation of primary} miRNA transcripts in $\mathrm{W}+\mathrm{W}$ and $\mathrm{W}+\mathrm{OS}$ treated leaves in wild type and ir-aoc. For each sample, one leaf on the rosette of a 32-day-old plants was treated with wounding plus water $(W+W)$ or wounding plus OS (W+OS) and harvested $1 \mathrm{~h}$ or $5 \mathrm{~h}$ post treatment. Untreated plants served as control (CTRL). Shown are mean ( \pm SE) levels of three replicates. Letters indicate significant differences $(P<0.05)$ in Fisher's PLSD test following an ANOVA.

Additional file 11: Time course expression of miRNAs and their targets in $\mathbf{W}+\mathbf{W}$ and $\mathbf{W}+\mathbf{O S}$ treated leaves. (A) qPCR data showing the abundance of mature miRNAs. (B) Microarray data showing the accumulation of miRNAs targets. For each sample, one leaf on the rosette of a 32-day-old WT plant was left untreated (control) or treated with wounding plus water $(\mathrm{W}+\mathrm{W})$ or wounding plus $\mathrm{OS}$

$(\mathrm{W}+\mathrm{OS})$ and harvested $1 \mathrm{~h}$ or $5 \mathrm{~h}$ post treatment. Shown are mean $( \pm \mathrm{SE})$ levels of three replicates per line. Lowercase letters (W+OS) and italic letters $(W+W)$ indicate significant differences $(P<0.05)$ in Fisher's PLSD tests following an ANOVA.

Additional file 12: Microarray data showing accumulation of primary miRNAs in $\mathrm{W}+\mathrm{W}$ and $\mathrm{W}+\mathrm{OS}$ treated leaves of $N$. attenuata. Mean ( \pm SE) levels of normalized transcript abundance of miRNAs and tasiRNAs target genes after $\mathrm{W}+\mathrm{W}$ and $\mathrm{W}+\mathrm{OS}$ treatments. Untreated plants served as control (CTRL). Signal intensities in microarray data was normalized using the $75^{\text {th }}$ percentile value and log2-transformation. Letters indicate significant differences $(P<0.05)$ in Fisher's PLSD test following an ANOVA

\section{Competing interests}

The authors declare that they have no competing interests.

\section{Authors' contributions}

TAB performed the experimental work and analyzed the data. TAB, ITB and SGK participated in the design of the study. ITB and SGK conceived of the study and edited the manuscript. TAB drafted the manuscript. All authors read and approved the final manuscript.

\section{Acknowledgments}

We thank Dr. Tamara Krügel and the gardening staff for growing and maintaining plants, Dr. Meredith C. Schuman for language editing, and the Max Planck Society for funding.

Received: 6 August 2012 Accepted: 4 November 2012 Published: 7 November 2012

\section{References}

1. Wu L, Zhou H, Zhang Q, Zhang J, Ni F, Liu C, Qi Y: DNA methylation mediated by a microRNA pathway. Mol Cell 2010, 38(3):465-475.
2. Ronemus M, Martienssen R: RNA interference: methylation mystery. Nature 2005, 433(7025):472-473

3. Chellappan P, Xia J, Zhou X, Gao S, Zhang X, Coutino G, Vazquez F, Zhang W, Jin $\mathrm{H}$ : siRNAs from miRNA sites mediate DNA methylation of target genes. Nucleic Acids Res 2010, 38(20):6883-6894.

4. Khraiwesh B, Arif MA, Seumel Gl, Ossowski S, Weigel D, Reski R, Frank W: Transcriptional control of gene expression by microRNAs. Cell 2010, 140(1):111-122.

5. Bartel DP: MicroRNAs: genomics, biogenesis, mechanism, and function. Cell 2004, 116(2):281-297.

6. Henderson IR, Zhang X, Lu C, Johnson L, Meyers BC, Green PJ, Jacobsen SE: Dissecting Arabidopsis thaliana DICER function in small RNA processing, gene silencing and DNA methylation patterning. Nat Genet 2006, 38(6):721-725.

7. Kurihara $Y$, Watanabe $Y$ : Arabidopsis micro-RNA biogenesis through Dicerlike 1 protein functions. Proc Natl Acad Sci USA 2004, 101(34):12753-12758.

8. Ruiz-Ferrer $V$, Voinnet $O$ : Roles of plant small RNAs in biotic stress responses. Annu Rev Plant Biol 2009, 60:485-510.

9. Rajagopalan $\mathrm{R}$, Vaucheret $\mathrm{H}$, Trejo J, Bartel DP: A diverse and evolutionarily fluid set of microRNAs in Arabidopsis thaliana. Genes Dev 2006, 20(24):3407-3425.

10. Xie Z, Allen E, Wilken A, Carrington JC: DICER-LIKE 4 functions in transacting small interfering RNA biogenesis and vegetative phase change in Arabidopsis thaliana. Proc Natl Acad Sci USA 2005, 102(36):12984-12989.

11. Marin E, Jouannet V, Herz A, Lokerse AS, Weijers D, Vaucheret H, Nussaume L, Crespi MD, Maizel A: miR390, Arabidopsis TAS3 tasiRNAs, and their AUXIN RESPONSE FACTOR targets define an autoregulatory network quantitatively regulating lateral root growth. Plant Cell 2010, 22(4):1104-1117.

12. Chen XM: Small RNAs and their roles in plant development. Annu Rev Cell Dev Bi 2009, 25:21-44.

13. Willmann MR, Mehalick AJ, Packer RL, Jenik PD: MicroRNAs regulate the timing of embryo maturation in Arabidopsis. Plant Physiol 2011, 155 (4):1871-1884

14. Kidner CA, Martienssen RA: Spatially restricted microRNA directs leaf polarity through ARGONAUTE1. Nature 2004, 428(6978):81-84.

15. Palatnik JF, Allen E, Wu X, Schommer C, Schwab R, Carrington JC, Weigel D: Control of leaf morphogenesis by microRNAs. Nature 2003, 425(6955):257-263.

16. Yoon EK, Yang JH, Lim J, Kim SH, Kim SK, Lee WS: Auxin regulation of the microRNA390-dependent transacting small interfering RNA pathway in Arabidopsis lateral root development. Nucleic Acids Res 2010, 38(4):1382-1391.

17. Liu Q, Chen YQ: Insights into the mechanism of plant development: interactions of miRNAs pathway with phytohormone response. Biochem Biophys Res Commun 2009, 384(1):1-5.

18. Schommer C, Palatnik JF, Aggarwal P, Chetelat A, Cubas P, Farmer EE, Nath $U$, Weigel D: Control of jasmonate biosynthesis and senescence by miR319 targets. PLOS Biol 2008, 6(9):e230.

19. Aukerman MJ, Sakai H: Regulation of flowering time and floral organ identity by a MicroRNA and its APETALA2-like target genes. Plant Cell 2003, 15(11):2730-2741.

20. Khraiwesh B, Zhu JK, Zhu J: Role of miRNAs and siRNAs in biotic and abiotic stress responses of plants. Biochim Biophys Acta 2012, 1819(2):137-148.

21. Kulcheski FR, de Oliveira LF, Molina LG, Almerao MP, Rodrigues FA, Marcolino J, Barbosa JF, Stolf-Moreira R, Nepomuceno AL, MarcelinoGuimaraes FC, et al: Identification of novel soybean microRNAs involved in abiotic and biotic stresses. BMC Genomics 2011, 12:307.

22. Xin M, Wang Y, Yao Y, Xie C, Peng H, Ni Z, Sun Q: Diverse set of microRNAs are responsive to powdery mildew infection and heat stress in wheat (Triticum aestivum L.). BMC Plant Biol 2010, 10:123.

23. Zhou L, Liu Y, Liu Z, Kong D, Duan M, Luo L: Genome-wide identification and analysis of drought-responsive microRNAs in Oryza sativa. J Exp Bot 2010, 61(15):4157-4168.

24. Zhou X, Wang G, Zhang W: UV-B responsive microRNA genes in Arabidopsis thaliana. Mol Syst Biol 2007, 3:103.

25. Chiou TJ, Aung K, Lin SI, Wu CC, Chiang SF, Su CL: Regulation of phosphate homeostasis by MicroRNA in Arabidopsis. Plant Cell 2006, 18(2):412-421.

26. Reyes $\lrcorner$, Chua $\mathrm{NH}$ : $\mathrm{ABA}$ induction of miR159 controls transcript levels of two MYB factors during Arabidopsis seed germination. Plant J 2007, 49(4):592-606 
27. Jones-Rhoades MW, Bartel DP: Computational identification of plant microRNAs and their targets, including a stress-induced miRNA. Mol Cell 2004, 14(6):787-799.

28. Tang S, Wang Y, Li Z, Gui Y, Xiao B, Xie J, Zhu QH, Fan L: Identification of wounding and topping responsive small RNAs in tobacco (Nicotiana tabacum). BMC Plant Biol 2012, 12(1):28.

29. Zhang W, Gao S, Zhou X, Chellappan P, Chen Z, Zhang X, Fromuth N, Coutino G, Coffey $\mathrm{M}$, Jin $\mathrm{H}$ : Bacteria-responsive microRNAs regulate plant innate immunity by modulating plant hormone networks. Plant Mol Biol 2011, 75(1-2):93-105.

30. Navarro L, Jay F, Nomura K, He SY, Voinnet O: Suppression of the microRNA pathway by bacterial effector proteins. Science 2008, 321(5891):964-967.

31. Kasschau KD, Xie Z, Allen E, Llave C, Chapman EJ, Krizan KA, Carrington JC: P1/ HC-Pro, a viral suppressor of RNA silencing, interferes with Arabidopsis development and miRNA function. Dev Cell 2003, 4(2):205-217.

32. Li H, Deng $Y$, Wu T, Subramanian S, Yu O: Misexpression of miR482, miR1512, and miR1515 increases soybean nodulation. Plant Physiol 2010, 153(4):1759-1770.

33. Schwachtje J, Baldwin IT: Why does herbivore attack reconfigure primary metabolism? Plant Physiol 2008, 146(3):845-851.

34. Kessler A, Baldwin IT: Herbivore-induced plant vaccination. Part I. The orchestration of plant defenses in nature and their fitness consequences in the wild tobacco Nicotiana attenuata. Plant J 2004, 38(4):639-649.

35. Kang JH, Wang L, Giri A, Baldwin IT: Silencing threonine deaminase and JAR4 in Nicotiana attenuata impairs jasmonic acid-isoleucine-mediated defenses against Manduca sexta. Plant Cell 2006, 18(11):3303-3320.

36. Halitschke R, Schittko U, Pohnert G, Boland W, Baldwin IT: Molecular interactions between the specialist herbivore Manduca sexta (Lepidoptera, Sphingidae) and its natural host Nicotiana attenuata. III. Fatty acid-amino acid conjugates in herbivore oral secretions are necessary and sufficient for herbivore-specific plant responses. Plant Physiol 2001, 125(2):711-717.

37. Bonaventure G, VanDoorn A, Baldwin IT: Herbivore-associated elicitors: FAC signaling and metabolism. Trends Plant Sci 2011, 16(6):294-299.

38. McCloud ES, Baldwin IT: Herbivory and caterpillar regurgitants amplify the wound-induced increases in jasmonic acid but not nicotine in Nicotiana sylvestris. Planta 1997, 203(4):430-435

39. Halitschke R, Ziegler J, Keinanen M, Baldwin IT: Silencing of hydroperoxide lyase and allene oxide synthase reveals substrate and defense signaling crosstalk in Nicotiana attenuata. Plant J 2004, 40(1):35-46.

40. Kallenbach M, Alagna F, Baldwin IT, Bonaventure G: Nicotiana attenuata SIPK, WIPK, NPR1, and fatty acid-amino acid conjugates participate in the induction of jasmonic acid biosynthesis by affecting early enzymatic steps in the pathway. Plant Physiol 2010, 152(1):96-106.

41. Paschold A, Halitschke R, Baldwin IT: Co(i)-ordinating defenses: NaCOI1 mediates herbivore- induced resistance in Nicotiana attenuata and reveals the role of herbivore movement in avoiding defenses. Plant $J$ 2007, 51(1):79-91.

42. Pandey SP, Shahi P, Gase K, Baldwin IT: Herbivory-induced changes in the small-RNA transcriptome and phytohormone signaling in Nicotiana attenuata. Proc Natl Acad Sci USA 2008, 105(12):4559-4564.

43. Liu Y, Gao Q, Wu B, Ai T, Guo X: NgRDR1, an RNA-dependent RNA polymerase isolated from Nicotiana glutinosa, was involved in biotic and abiotic stresses. Plant Physiol Biochem 2009, 47(5):359-368.

44. Pandey SP, Baldwin IT: RNA-directed RNA polymerase 1 (RdR1) mediates the resistance of Nicotiana attenuata to herbivore attack in nature. Plant J 2007, 50(1):40-53.

45. Bozorov TA, Prakash Pandey S, Dinh ST, Kim SG, Heinrich M, Gase K, Baldwin IT: DICER-like proteins and their role in plant-herbivore interactions in Nicotiana attenuata. J Integr Plant Biol 2012, 54(3):189-206.

46. Kim SG, Yon F, Gaquerel E, Gulati J, Baldwin IT: Tissue specific diurnal rhythms of metabolites and their regulation during herbivore attack in a native tobacco, Nicotiana attenuata. PLoS One 2011, 6(10):e26214.

47. Kallenbach M, Bonaventure G, Gilardoni PA, Wissgott A, Baldwin IT: Empoasca leafhoppers attack wild tobacco plants in a jasmonatedependent manner and identify jasmonate mutants in natural populations. Proc Natl Acad Sci USA 2012, 109(24):1548-1557.

48. Liang C, Zhang X, Zou J, Xu D, Su F, Ye N: Identification of miRNA from Porphyra yezoensis by high-throughput sequencing and bioinformatics analysis. PLoS One 2010, 5(5):e10698.
49. Frazier TP, Xie F, Freistaedter A, Burklew CE, Zhang B: Identification and characterization of microRNAs and their target genes in tobacco (Nicotiana tabacum). Planta 2010, 232(6):1289-1308

50. Allen E, Xie Z, Gustafson AM, Carrington JC: microRNA-directed phasing during trans-acting siRNA biogenesis in plants. Cell 2005, 121(2):207-221.

51. Shen D, Wang S, Chen H, Zhu QH, Helliwell C, Fan LJ: Molecular phylogeny of miR390-guided trans-acting siRNA genes (TAS3) in the grass family. Plant Syst Evol 2009, 283(1-2):125-132.

52. Adenot X, Elmayan T, Lauressergues D, Boutet S, Bouche N, Gasciolli V, Vaucheret $\mathrm{H}$ : DRB4-dependent TAS3 trans-acting siRNAs control leaf morphology through AGO7. Curr Biol 2006, 16(9):927-932.

53. Kim JH, Woo HR, Kim J, Lim PO, Lee IC, Choi SH, Hwang D, Nam HG Trifurcate feed-forward regulation of age-dependent cell death involving miR164 in Arabidopsis. Science 2009, 323(5917):1053-1057.

54. Schwachtje J, Minchin PE, Jahnke S, van Dongen JT, Schittko U, Baldwin IT: SNF1-related kinases allow plants to tolerate herbivory by allocating carbon to roots. Proc Natl Acad Sci USA 2006, 103(34):12935-12940.

55. Halitschke R, Baldwin IT: Antisense LOX expression increases herbivore performance by decreasing defense responses and inhibiting growthrelated transcriptional reorganization in Nicotiana attenuata. Plant $J$ 2003, 36(6):794-807

56. Luo QJ, Mittal A, Jia F, Rock CD: An autoregulatory feedback loop involving PAP1 and TAS4 in response to sugars in Arabidopsis. Plant Mol Biol 2012, 80(1):117-129.

57. Kaur H, Heinzel N, Schottner M, Baldwin IT, Galis I: R2R3-NaMYB8 regulates the accumulation of phenylpropanoid-polyamine conjugates, which are essential for local and systemic defense against insect herbivores in Nicotiana attenuata. Plant Physiol 2010, 152(3):1731-1747.

58. Onkokesung N, Gaquerel E, Kotkar H, Kaur H, Baldwin IT, Galis I: MYB8 controls inducible phenolamide levels by activating three novel hydroxycinnamoyl-coenzyme A:polyamine transferases in Nicotiana attenuata. Plant Physiol 2012, 158(1):389-407.

59. Schwab $R$, Voinnet $O$ : miRNA processing turned upside down. EMBO J 2009, 28(23):3633-3634.

60. Steffen P, Voss B, Rehmsmeier M, Reeder J, Giegerich R: RNAshapes: an integrated RNA analysis package based on abstract shapes. Bioinformatics 2006, 22(4):500-503.

61. Tamura K, Dudley J, Nei M, Kumar S: MEGA4: Molecular Evolutionary Genetics Analysis (MEGA) software version 4.0. Mol Biol Evol 2007, 24(8):1596-1599.

62. Molnar A, Schwach F, Studholme DJ, Thuenemann EC, Baulcombe DC: miRNAs control gene expression in the single-cell alga Chlamydomonas reinhardtii. Nature 2007, 447(7148):1126-1129.

63. Bubner B, Gase K, Baldwin IT: Two-fold differences are the detection limit for determining transgene copy numbers in plants by real-time PCR. BMC Biotechnol 2004, 4:14.

64. Wang L, Halitschke R, Kang JH, Berg A, Harnisch F, Baldwin IT: Independently silencing two JAR family members impairs levels of trypsin proteinase inhibitors but not nicotine. Planta 2007, 226(1):159-167.

\section{doi:10.1186/1471-2229-12-209}

Cite this article as: Bozorov et al.: Identification and profiling of miRNAs during herbivory reveals jasmonate-dependent and -independent patterns of accumulation in Nicotiana attenuata. BMC Plant Biology 2012 $12: 209$

\section{Submit your next manuscript to BioMed Central and take full advantage of:}

- Convenient online submission

- Thorough peer review

- No space constraints or color figure charges

- Immediate publication on acceptance

- Inclusion in PubMed, CAS, Scopus and Google Scholar

- Research which is freely available for redistribution 